\section{Preventing occupational stress in healthcare workers}

This is the abstract of a Cochrane Review published in the Cochrane Database of Systematic Reviews (CDSR) 2015, issue 4, art. $n^{\circ}$ CD002892. DOI: 10.1002/14651858.CD002892.pub5. For full text and details about the authors, see reference 1 .

\section{Jani H. Ruotsalainen, Jos H. Verbeek, Albert Mariné, Consol Serra}

The independent commentary was written by João Silvestre Silva-Junior

\section{ABSTRACT}

BACKGROUND: Healthcare workers can suffer from occupational stress which may lead to serious mental and physical health problems. OBJECTIVES: To evaluate the effectiveness of work and person-directed interventions in preventing stress at work in healthcare workers. METHODS:

Search methods: We searched the Cochrane Depression Anxiety and Neurosis Group trials Specialised Register, MEDLINE, PsychInfo and Cochrane Occupational Health Field database.

Selection criteria: Randomised controlled clinical trials (RCT) of interventions aimed at preventing psychological stress in healthcare workers. For work-directed interventions interrupted time series and prospective cohort were also eligible.

Data collection and analysis: Two authors independently extracted data and assessed trial quality. Meta-analysis and qualitative synthesis were performed where appropriate

MAIN RESULTS: We identified 14 RCTs, three cluster-randomised trials and two crossover trials, including a total of 1,564 participants in intervention groups and 1,248 controls. Two trials were of high quality. Interventions were grouped into 1) person-directed: cognitivebehavioural, relaxation, music-making, therapeutic massage and multicomponent; and 2) work-directed: attitude change and communication, support from colleagues and participatory problem solving and decision-making, and changes in work organisation. There is limited evidence that person-directed interventions can reduce stress (standardised mean difference or SMD -0.85; 95\% Cl -1.21, -0.49); burnout: Emotional Exhaustion (weighted mean difference or WMD -5.82; 95\% Cl $-11.02,-0.63$ ) and lack of Personal Accomplishment (WMD $-3.61 ; 95 \% \mathrm{Cl}-4.65,-2.58)$; and anxiety: state anxiety (WMD -9.42; 95\% $\mathrm{Cl}-16.92,-1.93)$ and trait anxiety (WMD $-6.91 ; 95 \% \mathrm{Cl}-12.80,-1.01)$. One trial showed that stress remained low a month after intervention (WMD $-6.10 ; 95 \% \mathrm{Cl}-8.44,-3.76)$. Another trial showed a reduction in Emotional Exhaustion (Mean Difference or MD -2.69; 95\% Cl -4.20,-1.17) and in lack of Personal Accomplishment (MD $-2.41 ; 95 \% \mathrm{Cl}-3.83,-0.99$ ) maintained up to two years when the intervention was boosted with refresher sessions. Two studies showed a reduction that was maintained up to a month in state anxiety (WMD $-8.31 ; 95 \% \mathrm{Cl}-11.49,-5.13)$ and trait anxiety (WMD -4.09; 95\% Cl -7.60,-0.58). There is limited evidence that work directed interventions can reduce stress symptoms (Mean Difference or MD -0.34; 95\% Cl -0.62,-0.06); Depersonalization (MD -1.14; 95\% Cl-2.18, -0.10 ), and general symptoms (MD $-2.90 ; 95 \% \mathrm{Cl}-5.16,-0.64)$. One study showed that the difference in stress symptom level was nonsignificant at six months (MD $-0.19 ; 95 \% \mathrm{Cl}-0.49,0.11)$.
AUTHORS' CONCLUSIONS: Limited evidence is available for the effectiveness of interventions to reduce stress levels in healthcare workers. Larger and better quality trials are needed.

The full text of this review is available free of charge from: http:// onlinelibrary.wiley.com/doi/10.1002/14651858.CD002892.pub5/epdf

\section{REFERENCE}

1. Ruotsalainen $J H$, Verbeek $J H$, Mariné A, Serra C. Preventing occupational stress in healthcare workers. Cochrane Database Syst Rev. 2015;4:CD002892.

\section{COMMENTS}

Occupational stress has been shown to be an important social determinant of health through its negative impact among healthcare workers.' Adverse conditions within the context and content of work are risk factors for problems such as decreased work ability, ${ }^{2}$ which may affect the quality and safety of healthcare services. Because of the socioeconomic impact of occupational stress, ${ }^{3}$ studies on interventions for minimizing its effects are necessary.

The Cochrane Occupational Safety and Health Review Group indicates in this review that the results relating to reduction of the effects of occupational stress through interventions at the individual level (such as cognitive-behavioral therapy or mental and physical relaxation) or at the organizational level (such as changes to work schedules) have been of limited extent. The authors indicate that further studies with greater representation of workers and validated methodologies are required, in order to advance the proposition of effective actions on this issue. Despite the results from this review, it is recommended that professionals responsible for promotion of overall health and prevention of disease among workers should undertake assessment and mitigation of this occupational hazard within the healthcare sector. After all, these missions are basic assumptions within Occupational Medicine and Occupational Health

\section{REFERENCES}

1. Weinberg A, Creed F. Stress and psychiatric disorder in healthcare professionals and hospital staff. Lancet. 2000;355(9203):533-7.

2. Martinez MC, do Rosário Dias de Oliveira Latorre M, Fischer FM. A cohort study of psychosocial work stressors on work ability among Brazilian hospital workers. Am J Ind Med. 2015;58(7):795-806.

3. Béjean S, Sultan-Taïeb H. Modeling the economic burden of diseases imputable to stress at work. Eur J Health Econ. 2015;6(1):16-23.

João Silvestre Silva-Junior. MD, MSc. Doctoral Student at University of São Paulo. Professor and Adjunct Coordinator of the Postgraduate Course on Occupational Medicine at Santa Casa Medical School. Current Scientific Director of Associação Paulista de Medicina do Trabalho and Scientific Coordinator of the Department of Occupational Medicine of Associação Paulista de Medicina. 